\title{
Effects of the Sijunzi decoction on the immunological function in rats with dextran sulfate-induced ulcerative colitis
}

\author{
WANGUI YU ${ }^{1}$, BING LU $^{1}$, HENGWEN ZHANG ${ }^{2}$, YANXIANG ZHANG $^{3}$ and JIN YAN ${ }^{1}$ \\ Departments of ${ }^{1}$ Physiology, ${ }^{2}$ Traditional Medicine and ${ }^{3}$ Laboratory, \\ Medical School of Yangtze University, Jingzhou, Hubei 434000, P.R. China
}

Received February 16, 2016; Accepted May 4, 2016

DOI: $10.3892 /$ br.2016.678

\begin{abstract}
The present study investigated the effects of the Sijunzi decoction (SJZD) at various dosages on the immunological function of rats with $3 \%$ dextran sulfate sodium (DSS; molecular weight 5,000)-induced ulcerative colitis (UC). A total of 40 male Wistar rats were randomly divided into 5 groups: Normal, model, low-dose SJZD, moderate-dose SJZD and high-dose SJZD groups. The $3 \%$ DSS was intragastrically administered for 7 consecutive days in order to induce the UC model. The normal group consumed distilled water. Subsequently, SJZD $(5.0,10.0$ and $30.0 \mathrm{~g} / \mathrm{kg}$ ) was intragastrically administered, and scores of the disease activity index (DAI) were calculated. After 2 weeks, all the rats were sacrificed. Scores of the colon mucosa damage index (CMDI) were evaluated; and secretory immunoglobulin A (sIgA) and interleukin-2 (IL-2) were measured in intestinal tissue by ELISA assays. The model group rats had ulcers, hyperemia and interstitial edema and infiltrated inflammatory cells. SJZD attenuated the severity of the gross lesions and reduced the histopathological injuries. Compared with the normal group, DAI and CMDI were significantly increased $(\mathrm{P}<0.01)$, and levels of determined $\operatorname{sIg} \mathrm{A}$ in the intestinal mucosa and IL-2 in the intestinal tissue were significantly decreased $(\mathrm{P}<0.05)$ in the model group. Compared with the model group, moderate and high doses of SJZD showed a restoration effect on all the aforementioned indexes, and the high dose was the most effective. In conclusion, SJZD can ameliorate inflammation in DSS-induced UC rats. The mechanism is most likely due to enhancing intestinal local immunity.
\end{abstract}

\section{Introduction}

Ulcerative colitis (UC) is a significant type of inflammatory bowel diseases (IBD), which is formed in the intestinal mucosa of ulcer and chronic nonspecific inflammation as a disease of

Correspondence to: Dr Wangui Yu, Department of Physiology, Medical School of Yangtze University, 1 Nanhuan Road, Jingzhou, Hubei 434000, P.R. China

E-mail: yuwangui999@sina.com

Key words: Sijunzi decoction, ulcerative colitis, intestinal mucosa immunity, secretory immunoglobulin A, interleukin-2 the digestive tract. Abdominal pain, diarrhea, mucus, pus and blood stool, and tenesmus are the main clinical manifestations. The course of the disease is long, as it is difficult to cure, and it is apparent that relapse is easy and canceration may occur (1-3). Epidemiological data suggest that the incidence of UC, both domestic and abroad, increases each year $(4,5)$. Immune, genetic, environmental and free radical damage factors are involved in the pathogenesis of UC, and in particular, the intestinal immune system has an important role (6). The Sijunzi decoction (SJZD) is the basic prescription for strengthening the spleen and replenishing qi, which has numerous pharmacological effects such as promoting digestion and absorption, regulating gastrointestinal motility, causing resistance to gastrointestinal mucosal injury and enhancing intestinal mucosal immunity $(7,8)$. However, the study of SJZD on the therapeutic effect of experimental colitis and intestinal immune function of the local effects remains to be elucidated. In the present study, dextran sulfate sodium (DSS; molecular weight 5,000)-induced rat models of experimental colitis were first exposed to several doses of SJZD, and subsequently the therapeutic effect of three different doses of SJZD were observed in UC rats, including the effect on the intestinal mucosa of secretory immunoglobulin A (slgA) and interleukin-2 (IL-2), in order to provide a theoretical basis for clinical practice.

\section{Materials and methods}

Materials. In total, 40 adult male specific-pathogen free Wistar rats (weight, $250 \pm 10 \mathrm{~g}$ ) were obtained from the Hubei Provincial Experimental Animal Research Center (Hubei China; Animal Center license no. SCDXK2008-005; animal qualified certificate no. 00003033). DSS was purchased from the Shanghai Bao Biotechnology Co., Ltd. (Shanghai, China). SJZD was composed of ginseng, atractylodes, tuckahoe and liquorice following a 10:9:9:6 ratio. The IL-2 ELISA kit was from Wuhan Biological Engineering Co., Ltd. (Wuhan, China) and the sIgA ELISA kit was from Beijing Sunbio Biological Medical Technology Co., Ltd. (Beijing, China). The Ethics Committee of Yangtze University (Jingzhou, Hubei, China) approved our study protocol.

\section{Methods}

Grouping and administration. The $40 \mathrm{Wistar}$ rats were randomly divided into 5 groups: Normal, model, low-dose 
Table I. Disease activity index scoring criteria.

\begin{tabular}{lccc}
\hline Score & $\begin{array}{r}\text { Decreased } \\
\text { weight, } \%\end{array}$ & $\begin{array}{c}\text { Stool } \\
\text { property }\end{array}$ & Hematochezia \\
\hline 0 & 0 & Normal & Normal \\
1 & $1-5$ & & Positive fecal occult blood \\
2 & $6-10$ & Loose & Gross blood stool \\
3 & $11-15$ & & \\
4 & $>15$ & Diarrhea & \\
\hline
\end{tabular}

Table II. Colon mucosa damage index scoring criteria.

\begin{tabular}{ll}
\hline Score & Macroscopic observation of colon characteristics \\
\hline 0 & $\begin{array}{l}\text { No damage } \\
\text { Mucosal congestion, edema, mucosal erosion or } \\
1\end{array}$ \\
2 & $\begin{array}{l}\text { ulceration } \\
\text { Mucosal congestion, edema, mucous membrane } \\
\text { rough, mild erosion or intestinal adhesion } \\
\text { Mucosal congestion, edema, moderate erosion and } \\
\text { ulcer formation, but the ulcer diameter was }<1 \mathrm{~cm}\end{array}$ \\
4 & $\begin{array}{l}\text { Mucosal congestion, edema, moderate erosion and } \\
\text { ulcer formation, but the ulcer diameter was }>1 \mathrm{~cm}\end{array}$ \\
\end{tabular}

Table III. Effect of SJZD on SIgA and IL-2 in intestinal mucosa of rats.

\begin{tabular}{lccc}
\hline Group & SJZD, g/kg & sIgA, ng/ml & IL-2, pg/ml \\
\hline Normal & 0 & $131.51 \pm 11.83$ & $147.35 \pm 22.16$ \\
Model & 0 & $78.35 \pm 14.26^{\mathrm{a}}$ & $67.73 \pm 11.37^{\mathrm{a}}$ \\
Low dose & 5 & $83.74 \pm 15.97^{\mathrm{b}}$ & $88.48 \pm 12.61^{\mathrm{b}, \mathrm{c}}$ \\
Moderate dose & 10 & $108.57 \pm 14.74^{\mathrm{b}, \mathrm{c}}$ & $119.26 \pm 21.38^{\mathrm{d}}$ \\
High dose & 30 & $121.53 \pm 11.59^{\mathrm{b}, \mathrm{c}}$ & $133.51 \pm 20.98^{\mathrm{d}}$ \\
\hline
\end{tabular}

${ }^{\mathrm{a}} \mathrm{P}<0.01$ and ${ }^{\mathrm{b}} \mathrm{P}<0.05$ vs. normal group; ${ }^{\mathrm{c}} \mathrm{P}<0.05$ and ${ }^{\mathrm{d}} \mathrm{P}<0.01$ vs. model group. Data are mean \pm standard deviation, $n=8$. SJZD, Sijunzi decoction; sIgA, secretory immunoglobulin A; IL-2, interleukin-2.

SJZD, moderate-dose SJZD and high-dose SJZD groups, with 8 rats in each group. The normal group consumed distilled water ad libitum, and the other groups consumed distilled water containing 3\% DSS ad libitum for 7 days to induce the rat model of UC. Subsequently, the normal and model groups were administered $5 \mathrm{ml}$ saline daily; and the low, moderate and high-dose SJZD groups were administered 5.0, 10.0 and $30.0 \mathrm{SJZD} \mathrm{g} / \mathrm{kg}$, respectively, as the treatment, once a day for 2 weeks.

Disease activity index (DAI) and colon mucosa damage index (CMDI) scores. All the rats were closely monitored during the course of the experiment, including the weight and the stool character, according to the standard (9), and the DAI

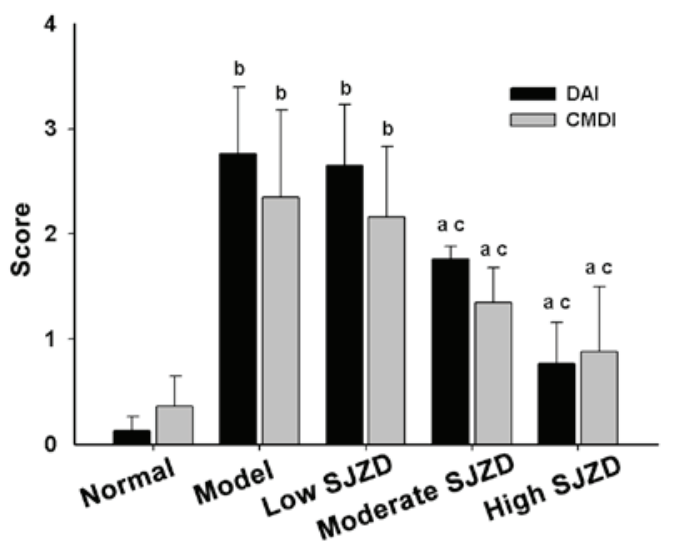

Figure 1. Effect of SJZD doses on the DAI and CMDI scores in rats. ${ }^{a} \mathrm{P}<0.05$ and ${ }^{\mathrm{b}} \mathrm{P}<0.01$ vs. normal group; ${ }^{\mathrm{C}} \mathrm{P}<0.05$ vs. model group. SJZD, Sijunzi decoction; DAI, disease activity index; CMDI, colon mucosa damage index.

score (Table I). All the animals were euthanized by intraperitoneal injection of $10 \%$ chloral hydrate. The distal $8 \mathrm{~cm}$ of the colon was excised, opened by longitudinal incision and rinsed with saline. Sections of the colon were fixed in $4 \%$ polyformaldehyde for histopathological evaluation of the CMDI score (Table II) (10). The remaining samples were stored at $-80^{\circ} \mathrm{C}$ for subsequent analyses.

ELISA. Rat intestine tissues (100 mg) were removed and mixed with $0.5 \mathrm{ml}$ phosphate-buffered saline ( $\mathrm{pH} 7.4)$, centrifuged at $6,000 \mathrm{x} \mathrm{g}$ for $40 \mathrm{~min}$, and the supernatants was removed for ELISA analysis to detect the levels of IL-2 and SIgA, as previously described (11).

Statistical analysis. Data are reported as the mean \pm standard deviation. Differences between treatment and control groups were analyzed by Student's unpaired t-test or analysis of variance followed by Tukey's post hoc test. $\mathrm{P}<0.05$ was considered to indicate a statistically significant difference. Statistics software SPSS 18.0 was used for statistical analyses (SPSS, Inc., Chicago, IL, USA).

\section{Results}

DAI and CMDI. The rats in the normal group had a normal diet and no apparent changes to body weight or defecation. The colonic mucosa was smooth with no adhesion, and the mucosal integrity was complete. There was no erosion or ulceration. Following the ingestion of $3 \%$ DSS, the rats in the other groups were listless with a shaggy appearance. There was a change in character of the stool, which was dark yellow dilute or semi dilute, and occult blood was positive on day 5 . By day 7, there was evident gross blood stool, movements were creeping or motionless, the rats ate less and there was weight loss. In the model group, mucosal congestion and edema of the colon were observed, and the ulcers were identified in the lesions. The ulcer surface epithelium was absent, the epithelium was damaged, there was inflammatory cell infiltration and the granulation tissue had formed. The epithelial repair was inadequate in the low-dose SJZD group, congestion remained and there was edema. In the moderate- or high-dose SJZD groups, the symptoms were relieved to different extents; 
A

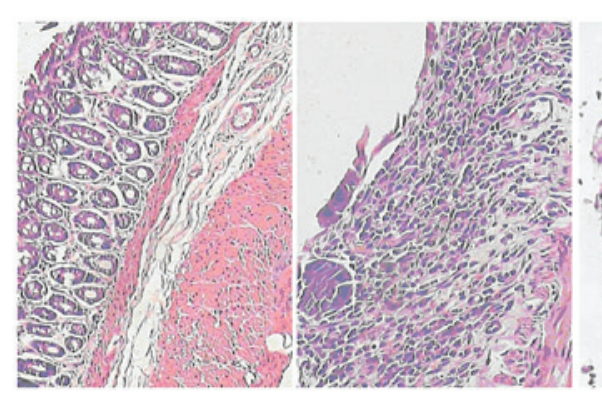

C

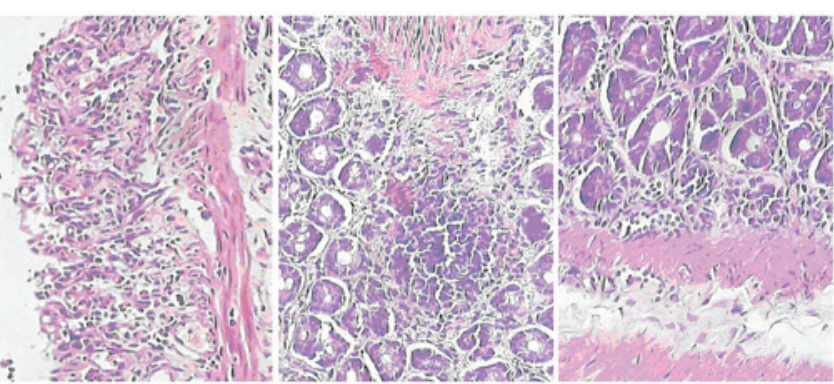

Figure 2. Pathological changes of the colon tissues. (A) Normal, (B) model, (C) low-dose, (D) moderate-dose and (E) high-dose SJZD groups.

diet and body weight were restored, and there were significant reductions in the number of cases of blood in the stool. After 2 weeks, mucous bloody stool was significantly reduced, feces were forming, ulcers were healing, and hyperemia and edema were significantly relieved. Compared with the normal group, DAI and CMDI were significantly higher in the model and low-dose SJZD groups compared to those in the normal group $(\mathrm{P}<0.01)$; however, in the moderate or high-dose SJZD groups the scores were significantly increased $(\mathrm{P}<0.05)$. Compared with the model group, the DAI and CMDI in the high-dose SJZD group were significantly decreased $(\mathrm{P}<0.05)$. No significant difference was observed between the low-dose SJZD and model groups $(\mathrm{P}>0.05)$. The changes of the DAI and CMDI scores in each group are shown in Fig. 1, and the changes to the structures are shown in Fig. 2.

sIgA content of intestinal mucosa. Compared with the normal group, the sIgA content in the intestinal mucosa of the model and SJZD groups were significantly decreased $(\mathrm{P}<0.01$ and $\mathrm{P}<0.05$, respectively). Compared with the model group, the sIgA content in the moderate- or high-dose SJZD groups were increased $(\mathrm{P}<0.05)$, and the level in the low-dose group was not significantly increased $(\mathrm{P}>0.05)$, as shown in Table III.

IL-2 content of intestinal mucosa. Compared with the normal group, the IL-2 level in the colonic homogenate of rats in the model and low-dose SJZD groups decreased significantly $(\mathrm{P}<0.01$ and $\mathrm{P}<0.05$, respectively). No significant difference in IL-2 level was observed for the moderate- and high-dose SJZD groups when compared to the normal group ( $P>0.05)$. When compared to the model group, the IL-2 level in the low-dose SJZD group and the moderate- and high-dose groups significantly increased $(\mathrm{P}<0.05$ and $\mathrm{P}<0.01$, respectively), as shown in Table III.

\section{Discussion}

DSS is one of the most common methods of the experimental UC model; the other drugs for the UC model include acetic acid, oxazolone and 2,4,6-trinitrobenzene sulfonic acid (12-14). DSS can damage the intestinal mucosal barrier function by inhibiting the proliferation of intestinal epithelial cells and injuring the immune dysfunction in the intestinal cavity, resulting in microflora disorders and the formation of UC $(15,16)$. The experimental process is simple and convenient, and the success rate is high. The present study established the UC model by DSS, and subsequently checked the success by the symptoms of the rats. DAI and CDMI indexes were significantly increased compared with the normal group, and colon pathological lesions and microscopic morphological changes were similar to the human UC. Following SJZD treatment, the DAI and CMDI indexes were decreased significantly, indicating that SJZD can effectively relieve the clinical symptoms of patients with UC, and reduce colon mucosal injury.

IBD are characterized by wasting and chronic intestinal inflammation triggered by various cytokine-mediated pathways $(17,18)$. sIgA has an important role in the intestinal mucosal immunity, which can neutralize viruses, toxins and enzymes and other biological active antigens, and produce a protective effect (19). IL-2 is an important immune regulatory factor, can promote T-cell growth and clone expansion, can induce or enhance the killing activity of cytotoxic cells, and also promotes B-cell proliferation, differentiation and antibody formation of biological activity $(20,21)$.

SJDZ is the basic prescription for strengthening the spleen and replenishing qi. Modern medical research has confirmed that this Chinese herbal medicine has the effect of reducing the inflammatory reaction of the intestinal mucosa, promoting the healing of an ulcer and repairing the tissue. Astragalus and ginseng improve the spleen qi, improve the immune function and increase the vitality of superoxide dismutase (22). Atractylodes can protect the spleen and reduce dampness, can enhance the reticuloendothelial system phagocytic function, can increase the rate of lymphocyte transformation, can enhance the cellular immune function, can elevate white blood cells and can evidently increase IgG content (23). The present study showed that SJZD can improve the content of $\operatorname{sg} \mathrm{A}$ in intestinal mucosa and the IL-2 level in the intestinal tissue.

In conclusion, SJZD can significantly improve the DSS-induced inflammatory response in rats with UC and its mechanism may be associated with the enhancement of the local immune response in the intestine.

\section{Acknowledgements}

The present study was supported by the Natural Science Foundation of Hubei province (grant no. 2010CDB04405) and Yangtze Youth Foundation (grant no. 2015cqn79). 


\section{References}

1. Hisamatsu T, Kanai T, Mikami Y, Yoneno K, Matsuoka K and Hibi T: Immune aspects of the pathogenesis of inflammatory bowel disease. Pharmacol Ther 137: 283-297, 2013.

2. Siegmund B: Management of mild-to-moderate ulcerative colitis. Dig Dis 33 (Suppl 1): 90-94, 2015.

3. Seo GS and Chae SC: Biological therapy for ulcerative colitis: An update. World J Gastroenterol 20: 13234-13238, 2014.

4. Jiang XL and Cui HF: An analysis of 10218 ulcerative colitis cases in China. World J Gastroenterol 8: 158-161, 2002.

5. Park SJ, Kim WH and Cheon JH: Clinical characteristics and treatment of inflammatory bowel disease: A comparison of Eastern and Western perspectives. World J Gastroenterol 20: 11525-11537, 2014

6. Russel MG: Changes in the incidence of inflammatory bowel disease: What does it mean? Eur J Intern Med 11: 191-196, 2000.

7. Liu L, Han L, Wong DY, Yue PY, Ha WY, Hu YH, Wang PX and Wong RN: Effects of Si-Jun-Zi decoction polysaccharides on cell migration and gene expression in wounded rat intestinal epithelial cells. Br J Nutr 93: 21-29, 2005.

8. Yu X, Cui Z, Zhou Z, Shan T, Li D and Cui N: Si-jun-zi decoction treatment promotes the restoration of intestinal function after obstruction by regulating intestinal homeostasis. Evid Based Complement Alternat Med 2014: 928579, 2014.

9. Wang X, Yang J, Cao Q and Tang J: Therapeutic efficacy and mechanism of water-soluble extracts of Banxiaxiexin decoction on BALB/c mice with oxazolone-induced colitis. Exp Ther Med 8: 1201-1204, 2014.

10. Myers KJ, Murthy S, Flanigan A, Witchell DR, Butler M, Murray S, Siwkowski A, Goodfellow D, Madsen K and Baker B: Antisense oligonucleotide blockade of tumor necrosis factor-alpha in two murine models of colitis. J Pharmacol Exp Ther 304: 411-424, 2003.

11. Katti MK: Assessment of serum IL-1, IL-2 and IFN- $\gamma$ levels in untreated pulmonary tuberculosis patients: Role in pathogenesis. Arch Med Res 42: 199-201, 2011.

12. Minaiyan M, Ghassemi-Dehkordi N, Mahzouni $\mathrm{P}$ and Ahmadi NS: Anti-inflammatory effect of Helichrysum oligocephalum DC extract on acetic acid - induced acute colitis in rats. Adv Biomed Res 3: 87, 2014.

13. Kaneko A, Kono T, Miura N, Tsuchiya N and Yamamoto M: Preventive effect of TU-100 on a type-2 model of colitis in mice: Possible involvement of enhancing adrenomedullin in intestinal epithelial cells. Gastroenterol Res Pract 2013: 384057, 2013.
14. Motavallian A, Minaiyan M, Rabbani M, Andalib S and Mahzouni P: Involvement of 5HT3 receptors in anti-inflammatory effects of tropisetron on experimental TNBS-induced colitis in rat. Bioimpacts 3: 169-176, 2013.

15. Okayama M, Tsubouchi R, Nishio H, Kato S and Takeuchi K: Protective effect of intra-rectal administration of rebamipide on dextran sulfate sodium-induced rat colitis. Digestion 70: 240-249, 2004

16. Randhawa PK, Singh K, Singh N and Jaggi AS: A review on chemical-induced inflammatory bowel disease models in rodents. Korean J Physiol Pharmacol 18: 279-288, 2014.

17. Owaga E, Hsieh RH, Mugendi B, Masuku S, Shih CK and Chang JS: Th17 cells as potential probiotic therapeutic targets in inflammatory bowel diseases. Int J Mol Sci 16: 20841-20858, 2015.

18. Tavakkoli H, Haghdani S, Adilipour H, Daghaghzadeh H, Minakari M, Adibi P, Ahmadi K and Emami MH: Serologic celiac disease in patients with inflammatory bowel disease. J Res Med Sci 17: 154-158, 2012.

19. Greenbaum E, Engelhard D, Levy R, Schlezinger M, Morag A and Zakay-Rones Z: Mucosal (SIgA) and serum (IgG) immunologic responses in young adults following intranasal administration of one or two doses of inactivated, trivalent anti-influenza vaccine. Vaccine 22: 2566-2577, 2004.

20. Liaw FP, Lau LC, Lim AS, Lim TH, Lee GY and Tien SL: CpG oligonucleotide and interleukin 2 stimulation enables higher cytogenetic abnormality detection rates than 12-o-tetradecanolyphorbol-13-acetate in Asian patients with B-cell chronic lymphocytic leukemia (B-CLL). Int J Hematol 100: 545-553, 2014.

21. Cho JH, Boyman O, Kim HO, Hahm B, Rubinstein MP, Ramsey C, Kim DM, Surh CD and Sprent J: An intense form of homeostatic proliferation of naive CD8+ cells driven by IL-2. J Exp Med 204: 1787-1801, 2007.

22. Yu DH, Bao YM, An LJ and Yang M: Protection of PC12 cells against superoxide-induced damage by isoflavonoids from Astragalus mongholicus. Biomed Environ Sci 22: 50-54, 2009.

23. Xie F, Sakwiwatkul K, Zhang C, Wang Y, Zhai L and Hu S: Atractylodis macrocephalae Koidz. polysaccharides enhance both serum IgG response and gut mucosal immunity. Carbohydr Polym 91: 68-73, 2013. 